Thursday 15 September 2011

\title{
Parallel Session B
}

Cardiovascular disease and mortality

OP21 MODELLING CORONARY HEART DISEASE MORTALITY IN NORTHERN IRELAND BETWEEN 1987 AND 2007

J Hughes, ${ }^{1^{*}} \mathrm{~F}$ Kee, ${ }^{1} \mathrm{~K}$ Bennett, ${ }^{2} \mathrm{M}$ O'Flaherty, ${ }^{3} \mathrm{~J}$ Critchley, ${ }^{4} \mathrm{M}$ Cupples,

$S$ Capewell ${ }^{3}$ Centre for Public Health, Queen's University, Belfast, UK; ${ }^{2}$ Trinity 
Centre for Health Sciences, St James's Hospital, Dublin, Ireland; ${ }^{3}$ Department of Public Health, University of Liverpool, UK; ${ }^{4}$ Division of Population Health Sciences and Education St George's, University of London, UK

\subsection{6/jech.2011.143586.21}

Purpose In 1987, Northern Ireland had one of the highest rates of Coronary heart disease (CHD) mortality in the world. Despite rapidly declining CHD mortality over the last two decades, CHD mortality in Northern Ireland are still among the highest in Europe. The purpose of this study was to use an epidemiological modelling approach to quantify how much of the mortality decline from 1987 to 2007 could be attributed to: (1) changes in population CHD risk factors and (2) medical and surgical treatments.

Methods The validated IMPACT CHD mortality model was used in all calculations to determine the number of deaths prevented or postponed (DPPs) for the period 1987-2007. For DPPs attributable to population risk factor changes, the calculation combined information on CHD deaths in 1987, prevalence of risk factor data from representative surveys (1987 and 2007) and regression coefficients (or RR) from the published literature which quantified the relationship between population changes for a specific risk factor and CHD mortality. For DPPs due to improved treatment uptake and effectiveness, the numbers of patients with CHD and their uptakes of specific treatments were obtained from local sources and combined with case death rates and $R R$ reductions due to treatment (both sourced from published literature). Sensitivity analyses were then carried out.

Results Between 1987 and 2007, the overall age-standardised CHD mortality in Northern Ireland (age 25-84 years) fell from 361 to 124 deaths per 100000 , resulting in some 3180 fewer CHD deaths in 2007. Changes in major cardiovascular risk factors accounted for approximately $60 \%$ of this decrease. Decreases in blood pressure, total cholesterol and smoking prevalence accounted for $74 \%$ of the mortality decline. However, these reductions were partially offset by adverse trends in diabetes, physical inactivity and obesity (combined $14 \%$ increase). Approximately $35 \%$ of the total mortality decrease was attributable to treatments, most notably secondary preventive therapies (15\%), treatments for Heart failure $(6 \%)$ and Acute Myocardial Infarction (5\%).

Conclusion CHD mortality in Northern Ireland decreased substantially between 1987 and 2007. Approximately 60\% of this fall was attributable to major cardiovascular risk factor changes, and approximately $35 \%$ was attributable to treatments. However, adverse trends in diabetes, obesity and physical inactivity are of major concern. To decrease future CHD deaths, more aggressive policies are therefore needed to control tobacco, promote healthy food and increase physical activity, as well as maximising the coverage of effective treatments. 